\title{
Crystal structure of dichlorodicyanoethyl(1,10-phenanthroline)tin, $\left[\left(\mathrm{NCCH}_{2} \mathrm{CH}_{2}\right)_{2} \mathrm{Sn}\right.$ (phen) $\left.\mathrm{Cl}_{2}\right]$
}

\author{
V. J. Hall and E. R. T. Tiekink \\ The University of Adelaide. Department of Chemistry, Australia 5005
}

Received October 10, 1997, CSD-No. 409124

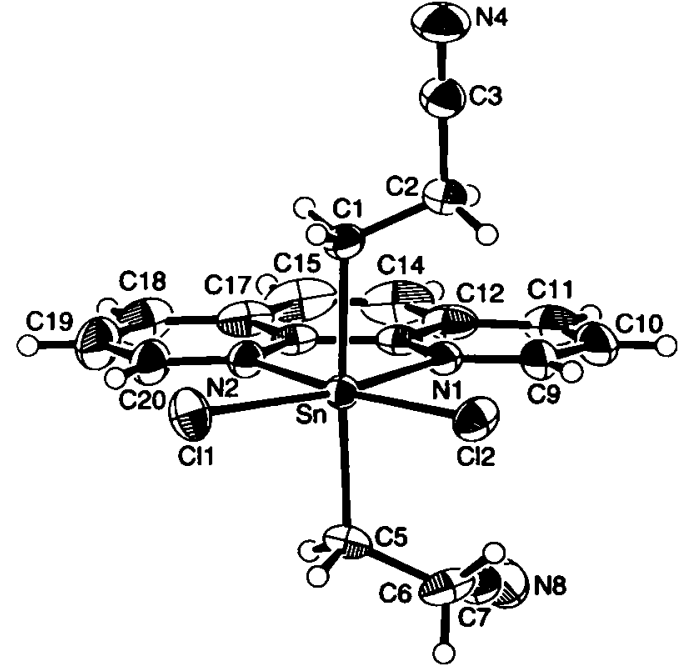

Source of material: Crystals were obtained from the reaction of [ $\left(\mathrm{NCCH}_{2} \mathrm{CH}_{2}\right)_{2} \mathrm{SnCl}_{2}$ ] (a gift from Prof. K. Jurkschat, Dortmund) and 1,10-phenanthroline in an acetonitrile/chloroform solution $(9 / 1 \mathrm{v} / \mathrm{v})$.

The $d(\mathrm{Sn}-\mathrm{Cl})$ and $d(\mathrm{Sn}-\mathrm{N})$ distances of $2.509(1) \AA, 2.549(1) \AA$, $2.355(2) \AA$ and 2.352 (2) $\AA$ are comparable with the $2,2^{\prime}$-bipyridyl analogue (see ref. 1), consistent with the similar coordinating potential of the two diimine bases.

$\mathrm{C}_{18} \mathrm{H}_{16} \mathrm{Cl}_{2} \mathrm{~N}_{4} \mathrm{Sn}$, triclinic, $P \overline{1}$ (No. 2), $a=10.237(1) \AA, b=12.194(2) \AA$, $c=8.235(3) \AA, \alpha=97.18(2)^{\circ}, \beta=107.87(2)^{\circ}, \gamma=77.02(1)^{\circ}$, $V=951.6 \AA^{3}, Z=2, R(F)=0.027, R_{w}(F)=0.030$.
Table 1. Parameters used for the X-ray data collection

$\begin{array}{ll}\text { Crystal: } & \text { colorless, block, size } 0.15 \times 0.19 \times 0.29 \mathrm{~mm} \\ \text { Wavelength: } & \text { Mo } K_{\alpha} \text { radiation }(0.7107 \AA) \\ \mu: & 16.31 \mathrm{~cm}^{-1} \\ \text { Diffractometer: } & \text { Rigaku AFC6R } \\ \text { Scan mode: } & \omega / 2 \theta \\ T_{\text {measurement: }} & 293 \mathrm{~K} \\ 2 \theta_{\max }: & 55^{\circ} \\ \mathrm{N}(\text { hk })_{\text {unique: }} & 3602 \\ \text { Criterion for } I_{0}: & I_{0}>3 \sigma\left(I_{0}\right) \\ \text { N(param) } & 226 \\ \text { Programs: } & \text { SHELXS-86, TEXSAN }\end{array}$

Table 2. Final atomic coordinates and displacement parameters (in $\AA^{2}$ )

\begin{tabular}{llllll}
\hline Atom & Site & \multicolumn{1}{c}{$x$} & \multicolumn{1}{c}{$y$} & $z$ & $U_{\text {iso }}$ \\
\hline H(1a) & $2 i$ & -0.0235 & -0.4237 & 0.2045 & 0.056 \\
H(1b) & $2 i$ & 0.1214 & -0.3912 & 0.2197 & 0.056 \\
H(2a) & $2 i$ & 0.1138 & -0.2935 & 0.4854 & 0.069 \\
H(2b) & $2 i$ & -0.0209 & -0.3415 & 0.4714 & 0.069 \\
H(5a) & $2 i$ & -0.2728 & -0.0676 & -0.0658 & 0.059 \\
H(5b) & $2 i$ & -0.1271 & -0.0388 & -0.0558 & 0.059 \\
H(6a) & $2 i$ & -0.1364 & 0.0385 & 0.2367 & 0.077 \\
H(6b) & $2 i$ & -0.2087 & 0.1133 & 0.0769 & 0.077 \\
H(9) & $2 i$ & -0.1283 & -0.1017 & 0.4920 & 0.063 \\
H(10) & $2 i$ & -0.2982 & -0.0715 & 0.6413 & 0.082 \\
H(11) & $2 i$ & -0.5022 & -0.1433 & 0.5225 & 0.081 \\
H(14) & $2 i$ & -0.648 & -0.2554 & 0.2786 & 0.086 \\
H(15) & $2 i$ & -0.6713 & -0.3570 & 0.0214 & 0.086 \\
H(18) & $2 i$ & -0.5826 & -0.4258 & -0.2368 & 0.083 \\
H(19) & $2 i$ & -0.4115 & -0.4367 & -0.3715 & 0.084 \\
H(20) & $2 i$ & -0.2170 & -0.3558 & -0.2372 & 0.068 \\
& & & & &
\end{tabular}

Table 3. Final atomic coordinates and displacement parameters (in $\AA^{2}$ )

\begin{tabular}{|c|c|c|c|c|c|c|c|c|c|c|}
\hline Atom & Site & $x$ & $y$ & $z$ & $U_{11}$ & $U_{22}$ & $U_{33}$ & $U_{12}$ & $U_{13}$ & $U_{23}$ \\
\hline Sn & $2 i$ & $-0.07613(2)$ & $-0.21372(2)$ & $0.12320(3)$ & $0.0336(1)$ & $0.0340(1)$ & $0.0345(1)$ & $-0.00620(7)$ & $0.01007(7)$ & $0.00274(7)$ \\
\hline $\mathrm{Cl}(1)$ & $2 i$ & $0.0265(1)$ & $-0.27918(9)$ & $-0.1208(1)$ & $0.0572(5)$ & $0.0823(7)$ & $0.0511(5)$ & $-0.0122(5)$ & $0.0290(4)$ & $-0.0036(5)$ \\
\hline $\mathrm{Cl}(2)$ & $2 i$ & $0.10525(9)$ & $-0.11180(7)$ & $0.3322(1)$ & $0.0478(4)$ & $0.0483(5)$ & $0.0651(6)$ & $-0.0172(4)$ & $0.0052(4)$ & $-0.0035(4)$ \\
\hline$N(1)$ & $2 i$ & $-0.2317(3)$ & $-0.1872(2)$ & $0.2906(3)$ & $0.047(1)$ & $0.033(1)$ & $0.039(1)$ & $-0.002(1)$ & $0.018(1)$ & $0.005(1)$ \\
\hline $\mathrm{N}(2)$ & $2 i$ & $-0.2678(3)$ & $-0.2989(2)$ & $-0.0235(3)$ & $0.040(1)$ & $0.038(1)$ & $0.040(1)$ & $-0.010(1)$ & $0.007(1)$ & $0.001(1)$ \\
\hline $\mathrm{N}(4)$ & $2 i$ & $0.2256(4)$ & $-0.5374(3)$ & $0.5798(5)$ & $0.079(2)$ & $0.062(2)$ & $0.083(3)$ & $0.005(2)$ & $-0.003(2)$ & $0.029(2)$ \\
\hline$N(8)$ & $2 i$ & $-0.4477(4)$ & $0.0784(3)$ & $0.1957(5)$ & $0.083(3)$ & $0.082(3)$ & $0.068(2)$ & $0.016(2)$ & $0.029(2)$ & $0.014(2)$ \\
\hline$C(1)$ & $2 i$ & $0.0336(3)$ & $-0.3669(3)$ & $0.2480(4)$ & $0.050(2)$ & $0.035(2)$ & $0.048(2)$ & $-0.003(1)$ & $0.006(1)$ & $0.005(1)$ \\
\hline$C(2)$ & $2 i$ & $0.0662(4)$ & $-0.3561(3)$ & $0.4415(5)$ & $0.071(2)$ & $0.046(2)$ & $0.049(2)$ & $-0.001(2)$ & $0.009(2)$ & $0.012(2)$ \\
\hline $\mathrm{C}(3)$ & $2 i$ & $0.1556(4)$ & $-0.4589(3)$ & $0.5198(5)$ & $0.060(2)$ & $0.054(2)$ & $0.053(2)$ & $-0.010(2)$ & $0.006(2)$ & $0.010(2)$ \\
\hline $\mathrm{C}(5)$ & $2 i$ & $-0.1829(3)$ & $-0.0563(3)$ & $0.0102(5)$ & $0.050(2)$ & $0.048(2)$ & $0.052(2)$ & $-0.005(1)$ & $0.013(2)$ & $0.021(2)$ \\
\hline$C(6)$ & $2 i$ & $-0.2106(4)$ & $0.0471(3)$ & $0.1297(6)$ & $0.061(2)$ & $0.034(2)$ & $0.092(3)$ & $-0.003(2)$ & $0.015(2)$ & $0.013(2)$ \\
\hline$C(7)$ & $2 i$ & $-0.3437(4)$ & $0.0644(3)$ & $0.1672(5)$ & $0.067(2)$ & $0.043(2)$ & $0.056(2)$ & $0.006(2)$ & $0.008(2)$ & $0.007(2)$ \\
\hline
\end{tabular}


Table 3. (Continued)

\begin{tabular}{|c|c|c|c|c|c|c|c|c|c|c|}
\hline Atom & Site & $x$ & $y$ & $z$ & $U_{11}$ & $U_{22}$ & $U_{33}$ & $U_{12}$ & $U_{13}$ & $U_{23}$ \\
\hline $\mathrm{C}(9)$ & $2 i$ & $-0.2134(4)$ & $-0.1312(3)$ & $0.4415(4)$ & $0.071(2)$ & $0.046(2)$ & $0.040(2)$ & $0.000(2)$ & $0.020(2)$ & $0.002(1)$ \\
\hline$C(10)$ & $2 i$ & $-0.3133(5)$ & $-0.1134(4)$ & $0.5313(5)$ & $0.107(4)$ & $0.061(3)$ & $0.051(2)$ & $0.015(2)$ & $0.041(2)$ & $0.010(2)$ \\
\hline$C(11)$ & $2 i$ & $-0.4314(5)$ & $-0.1559(4)$ & $0.4625(6)$ & $0.093(3)$ & $0.061(3)$ & $0.090(3)$ & $0.021(2)$ & $0.067(3)$ & $0.029(2)$ \\
\hline$C(12)$ & $2 i$ & $-0.4531(4)$ & $-0.2171(3)$ & $0.3041(5)$ & $0.051(2)$ & $0.049(2)$ & $0.080(3)$ & $0.010(2)$ & $0.035(2)$ & $0.027(2)$ \\
\hline$C(13)$ & $2 i$ & $-0.3493(3)$ & $-0.2296(2)$ & $0.2210(4)$ & $0.037(2)$ & $0.035(2)$ & $0.054(2)$ & $0.005(1)$ & $0.017(1)$ & $0.019(1)$ \\
\hline$C(14)$ & $2 i$ & $-0.5750(4)$ & $-0.2653(4)$ & $0.2219(8)$ & $0.048(2)$ & $0.072(3)$ & $0.144(5)$ & $0.007(2)$ & $0.054(3)$ & $0.043(3)$ \\
\hline$C(15)$ & $2 i$ & $-0.5895(4)$ & $-0.3237(4)$ & $0.0707(8)$ & $0.040(2)$ & $0.063(3)$ & $0.146(5)$ & $-0.005(2)$ & $0.025(3)$ & $0.036(3)$ \\
\hline$C(16)$ & $2 i$ & $-0.3677(3)$ & $-0.2901(2)$ & $0.0565(4)$ & $0.033(1)$ & $0.031(1)$ & $0.058(2)$ & $-0.002(1)$ & $0.008(1)$ & $0.014(1)$ \\
\hline$C(17)$ & $2 i$ & $-0.4873(4)$ & $-0.3376(3)$ & $-0.0189(6)$ & $0.041(2)$ & $0.046(2)$ & $0.086(3)$ & $-0.010(2)$ & $0.005(2)$ & $0.023(2)$ \\
\hline$C(18)$ & $2 i$ & $-0.5010(4)$ & $-0.3929(4)$ & $-0.1805(7)$ & $0.055(2)$ & $0.057(2)$ & $0.098(4)$ & $-0.025(2)$ & $-0.015(2)$ & $0.007(2)$ \\
\hline$C(19)$ & $2 i$ & $-0.4024(5)$ & $-0.3987(4)$ & $-0.2589(6)$ & $0.078(3)$ & $0.068(3)$ & $0.064(3)$ & $-0.029(2)$ & $-0.005(2)$ & $-0.011(2)$ \\
\hline$C(20)$ & $2 i$ & $-0.2869(4)$ & $-0.3509(3)$ & $-0.1782(5)$ & $0.058(2)$ & $0.053(2)$ & $0.051(2)$ & $-0.016(2)$ & $0.004(2)$ & $-0.005(2)$ \\
\hline
\end{tabular}

Acknowledgments. V. J. H. was the holder of a Commonwealth of Australia Postgraduate Research Award. The Australian Research Council is thanked for support.

\section{References}

1. Hall, V. J.; Tiekink, E. R. T.: Crystal structure of $2,2^{\prime}$-bipyridyldichlorodicyanoethyltin, [( $\left.\mathrm{NCCH}_{2} \mathrm{CH}_{2}\right)_{2} \mathrm{Sn}($ bipy $) \mathrm{Cl}_{2}$ ]. Z. Kristallogr. NCS 213 (1998) 403-404

2. Sheldrick, G. M.: SHELXS-86. Program for the automatic solution of crystal structure. University of Göttingen, Germany 1986.

3. TEXSAN: Single crystal structure analysis software. Version 1.7.1. Molecular Structure Corporation. The Woodlands, TX. USA 1995. 